\title{
What makes a good query? Prospects for a comprehensive theory of human information acquisition
}

\author{
Björn Meder ${ }^{1,2, \star}$, Vincenzo Crupi ${ }^{3}$, and Jonathan D. Nelson ${ }^{2,4}$ \\ ${ }^{1}$ Health and Medical University Potsdam, Germany \\ ${ }^{2}$ Max Planck Institute for Human Development, Germany \\ ${ }^{3}$ University of Turin, Italy \\ ${ }^{4}$ University of Surrey, Guildford, UK
}

\begin{abstract}
Searching for information in a goal-directed manner is central for learning, diagnosis, and prediction. Children ask questions to learn new concepts, doctors conduct medical tests to diagnose their patients, and scientists perform experiments to test their theories. But what makes a good query? What principles govern human information acquisition and how do people decide which query to conduct to achieve their goals? What challenges need to be met to advance theory and psychology of human inquiry? Addressing these issues, we introduce the conceptual and mathematical ideas underlying different models of the value of information, what purpose these models serve in psychological research, and how they can be integrated in a unified computational framework. We also discuss the conflict between short- and long-term efficiency of prominent methods for query selection, and the resulting normative and methodological implications for studying human sequential search. A final point of discussion concerns the relations between probabilistic (Bayesian) models of the value of information and heuristic search strategies, and the insights than can be gained from bridging different levels of analysis and types of models. We conclude by discussing open questions and challenges that research needs to address to build a comprehensive theory of human information acquisition.
\end{abstract}

Keywords: search; information acquisition; optimal experimental design (OED); entropy; value of information; stepwise methods, heuristics

\section{The Psychology of Human Information Acquisition}

Searching for relevant information to support learning and reasoning is central to intelligent and goal-directed behavior. Cognitive development is guided by children's ability to actively acquire information about their physical and social environment. Doctors routinely perform tests to diagnose their patients. And, of course, scientists conduct experiments to test their theories and hypotheses.

Psychological research on how humans acquire information in a self-directed manner began in the wake of the cognitive revolution (Bruner et al., 1956; Mosher \& Hornsby, 1966; Wason, 1960). Many of these earlier studies were inspired by Popper's (1959) philosophy of science and his method of falsification: that from a logical point of view scientific theories cannot be proven to be true, but they can be shown to be wrong if their predictions are inconsistent with the outcome of an experiment. Accordingly, queries (e.g., questions, tests, experiments) are only useful if they can yield data that could potentially falsify a hypothesis.

Adopting a logical framework and falsificationism as the normatively correct approach to information acquisition, psychologists devised empirical studies to find out whether people would intuitively seek out potentially disconfirming evidence. A prominent example is the selection task, in which participants can acquire information to test whether a conditional rule holds (Wason, 1968). Presented with four cards and a rule like "If there is a vowel on one side of any card, then there is an even number on its other side," searchers could turn over one or multiple cards to attempt to falsify the rule. Contrary to the prescriptions of a logico-deductive method, and paralleling findings from related tasks (Wason, 1960), few participants selected queries in accordance with a falsificationist strategy. In line with the emerging heuristics-and-biases program (Kahneman \& Tversky, 1974), Wason and others suggested that people's hypothesis-testing strategies are prone to a "confirmation bias" and other suboptimalities (Klayman \& Ha, 1987; Nelson \& McKenzie, 2009; Nickerson, 1996).

\footnotetext{
* Correspondence concerning this article should be addressed to Björn Meder, Health and Medical University Potsdam, Olympischer Weg 1, 14471 Potsdam, Germany. Email: bjoern.meder $@$ health-and-medical-university.de or meder@mpib-berlin.mpg.de.
} 
Around the 1980s, psychologists started using a different theoretical framework for conceptualizing and evaluating human search, inspired by approaches developed in information theory (Shannon, 1948), philosophy of science (Good, 1950), statistics (Lindley, 1956), and decision theory (Savage, 1954). A key idea in this framework, foreshadowed by Chamberlin's (1890) method of multiple working hypotheses, is that the goal of scientific information acquisition is to discriminate among multiple possible hypotheses, rather than to falsify a single hypothesis. Commonly referred to as the Optimal Experimental Design (OED) framework, query selection is conceptualized as probabilistic inductive inference, where the outcome of a query is used to revise beliefs about the considered hypotheses. Updating beliefs in light of new data provides a certain amount of information, with different ways of measuring this quantity in relation to a searcher's beliefs and goals. These models have informed theory and empirical research on central issues in perception, developmental and cognitive psychology, and neuroscience.

Here, we discuss four key questions. First, how can we formalize the value of information and how do different models differ conceptually and mathematically? Second, how can these models support the theoretical and empirical analysis of human information acquisition? Third, what normative and computational principles govern sequential search, and how can the tension between short- and longrun optimality inform research on people's ability to conduct an efficient series of queries? Fourth, what are the relations between probabilistic (Bayesian) models of the value of information and heuristic approaches to information acquisition, and what insights can be gained from bridging different levels of analysis? We conclude by discussing current challenges and prospects for a comprehensive theory of human information search.

\section{What Makes a Good Query?}

How can we quantify the informational value of a query - a verbal question, a medical test, an eye movement, or an experiment? Several models to address this issue have been proposed in different fields, including information theory, philosophy of science, statistics, and psychology. Placed within the broad OED framework, these models evaluate queries according to different conceptual ideas and corresponding measures, such as the expected reduction in uncertainty (Lindley, 1956) or the expected improvement in classification accuracy (Baron, 1985). The quantities underpinning OED models (e.g., entropy measures) are widely used, including applications in ecology (Crupi, 2019; Simpson, 1949), economics (Gini, 1921), machine learning (Settles, 2010), and physics (Beck, 2005). They also provide the foundation of active learning strategies used to adaptively sample high-dimensional search spaces, for instance in material sciences (Lookman et al., 2019), drug discovery (Murphy, 2011), or learning of protein interactions (Mohamed et al., 2010). How humans search large decision spaces has also been addressed in recent psychological research (Meder et al., 2021; Schulz et al., 2019; Wu et al., 2018).

In cognitive science, OED models serve as descriptive or normative models for assessing human information acquisition in different domains (for reviews see Coenen et al., 2019; Crupi et al., 2018; Nelson, 2005; Gureckis \& Markant, 2012). Key applications include perceptual tasks (Najemnik \& Geisler, 2005; Nelson \& Cottrell, 2007), categorization (Markant \& Gureckis, 2014; Nelson et al., 2010) associative learning (Kruschke, 2008), causal induction (Bramley et al., 2015; Steyvers et al., 2003), and hypothesis testing (Austerweil \& Griffiths, 2011; Crupi et al., 2009; Oaksford \& Chater, 1994, 1996). OED principles are also frequently used in developmental research (Kachergis et al., 2016; Nelson et al., 2014; Ruggeri \& Lombrozo, 2015; Ruggeri et al., 2015) and neuroscience (Filimon et al., 2020; Nakamura, 2006). Applied issues include eye witness identification (Wells \& Lindsay, 1980), medical diagnosis (Benish, 1999), reading (Legge et al., 1997), and the design of psychological experiments (Myung \& Pitt, 2009).

\section{Optimal Experimental Design (OED): Probabilistic Models of the Value of Information}

OED models provide a formal account of the epistemic value of information and the usefulness of possible queries. Typically, OED models are defined solely in terms of the relevant probability model, and are therefore most adequate for situations where the goal of the searcher is purely epistemic. Accordingly, OED models provide informational (or epistemic) utility functions. 
The general method for evaluating a query $Q$ based on OED principles requires specifying the prior probabilities of the hypotheses, the likelihood of each query outcome given the hypotheses, and a measure of the epistemic utility of each outcome. For instance, the information value of a medical test (Benish, 1998) that can come out positive or negative is fully specified by the prior probabilities of the condition (e.g., prior probability of the disease, for instance derived from epidemiological data), and the likelihoods of a positive test given that the medical condition is present or absent, respectively. Other examples are classification tasks, where different features can be queried before making a prediction (Nelson et al., 2010), or visual search tasks, where eye movements must be directed towards informative parts of a scene to find a target item (Najemnik \& Geisler, 2005).

Based on the probability model, a pre-posterior analysis (Raiffa \& Schlaifer, 1961) can be conducted, where the amount of obtained information is quantified by comparing the prior probability distribution over the hypotheses before asking query $Q$ with the posterior distribution after asking $Q$ and obtaining outcome $q_{j}$. (Capital letters refer to random variables, such as a query $Q$, whose answer is not yet known. Lowercase variables refer to known values; for instance, $q_{j}$ is a specific obtained answer.) If a query $Q$ has $m$ possible answers, then its expected informational utility, $e u(Q)$, is the weighted average usefulness of its possible answers:

$$
e u(Q)=\sum_{j=1}^{m} P\left(q_{j}\right) u\left(q_{j}\right)
$$

where $P\left(q_{j}\right)$ denotes the probability of observing outcome $q_{j}$ and $u\left(q_{j}\right)$ denotes its information value.

Figure 1 illustrates the basic rationale of determining queries' information value using a searchand-classification task with a binary hypothesis space (species A vs. species B) and two binary features, the specimen's "eye" and "claw" (Nelson et al., 2010). The probabilistic structure of the environment is shown in Figure 1a, that is, the hypotheses' prior probabilities, $P\left(h_{i}\right)$, and the individual feature likelihoods $P\left(q_{j} \mid h_{i}\right)$ (numbers from Wu et al., 2017, Experiment 1). The goal is to classify the specimen as species A or B, after querying one of the two features (Figure 1b). Each query could yield two outcomes (feature values) with varying implications for the hypotheses' posterior probabilities, i.e., for each $P\left(h_{i} \mid q_{j}\right)$. But how exactly can we use these quantities to determine the information value of the two queries?

Many different value-of-information models within the broad OED framework have been proposed for quantifying the value of queries. These models can make strongly diverging claims about the informational utility of a query (Nelson, 2005). Here, we briefly introduce some prominent entropybased models of the value of information, which constitute the most widely used class of measures across different disciplines. Importantly, there are deep mathematical relationships among seemingly different models that allow them to be treated in a unified mathematical framework (Crupi et al., 2018; Sharma \& Mittal, 1975).

\subsection{Information Gain: Reducing Uncertainty}

Information gain (IG; Lindley, 1956) values queries in accordance with the expected reduction in uncertainty, where uncertainty is measured via Shannon (1948) entropy. The model contrasts the entropy in the hypothesis space before conducting a query with the expected entropy after the query, with the information value of a query being the expected amount of entropy reduction.

The Shannon entropy of a random variable $H$, ent $(H)$, corresponding to the true hypothesis (e.g., the species in Figure 1), is defined as

$$
\operatorname{ent}_{\text {Shannon }}(H)=\sum_{i=1}^{n} P\left(h_{i}\right) \log \frac{1}{P\left(h_{i}\right)}
$$

where there are $n$ possible hypotheses $h_{1}, h_{2}, \ldots, h_{n}$. (The choice of base of logarithm is arbitrary; here, we use $\log _{2}$ such that IG is measured in bits; Figure 2a.) The corresponding informational utility of query outcome $q_{j}$ is 
a) Task environment

Feature configurations

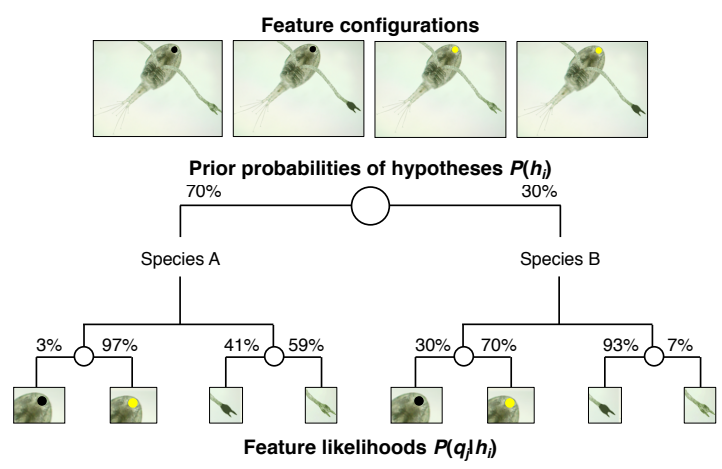

b) Search task

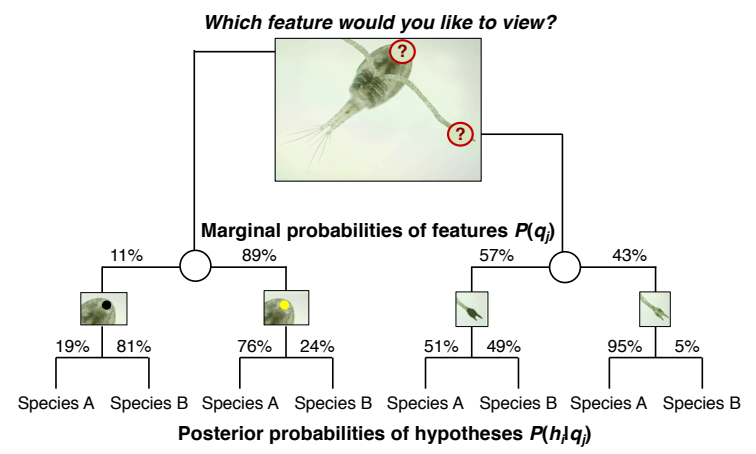

c) Informational utilities of queries and outcomes

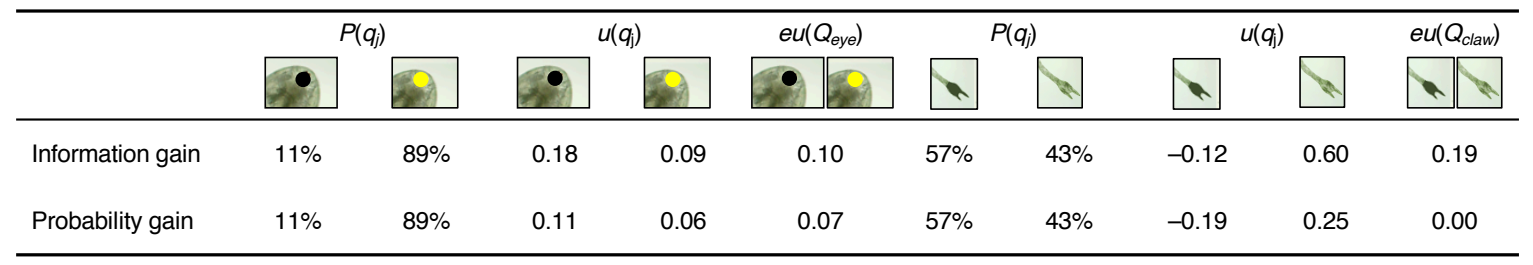

Fig. 1. A search-and-classification task with a binary hypothesis (species A vs. B) and two binary features (the specimens' "eye" and "claw" feature, Nelson et al., 2010). a) Task structure, i.e., the hypotheses' prior probabilities $P\left(h_{i}\right)$, and the feature likelihoods, $P\left(q_{j} \mid h_{i}\right)$. b) Information search task, where one of the two features can be queried before making a classification decision. The tree shows the marginal probabilities of the feature values, $P\left(q_{j}\right)$, and the posterior probabilities of the hypotheses given each feature value, $P\left(h_{i} \mid q_{j}\right)$. c) Informational utilities of outcomes, $u\left(q_{j}\right)$, and queries, $e u(Q)$, according to information gain and probability gain. Information gain considers querying the claw feature more useful than querying the eye feature, $e u\left(Q_{\text {eye }}\right)<e u\left(Q_{\text {claw }}\right)$, whereas probability gain considers the eye feature more informative, $e u\left(Q_{\text {eye }}\right)>e u\left(Q_{\text {claw }}\right)$.

$$
u_{I G}\left(q_{j}\right)=\sum_{i=1}^{n} P\left(h_{i}\right) \log \frac{1}{P\left(h_{i}\right)}-P\left(h_{i} \mid q_{j}\right) \log \frac{1}{P\left(h_{i} \mid q_{j}\right)}
$$

where the first term corresponds to the prior entropy, ent $(H)$, and the second term denotes the expected posterior entropy of $H$, given answer $q_{j}$. Figure 1c shows the IG of the individual query outcomes, $u\left(q_{j}\right)$ and the informational utility of the two queries, $e u\left(Q_{\text {eye }}\right)$ and $e u\left(Q_{\text {claw }}\right)$, given by the weighted average epistemic utility of the outcomes (Equation 1). According to IG, querying the claw feature is more useful than querying the eye feature, eu $\left(Q_{\text {eye }}\right)=0.1$ bits vs. eu $\left(Q_{\text {claw }}\right)=0.19$ bits.

IG is probably the most-widely used measure for quantifying uncertainty reduction in information acquisition. Applications in psychology range from hypothesis testing (Austerweil \& Griffiths, 2011; Nelson et al., 2001; Oaksford \& Chater, 1994, 1996) and causal induction (Bramley et al., 2015; Steyvers et al., 2003) to the analysis of eye movements (Legge et al., 1997; Najemnik \& Geisler, 2005; Nelson \& Cottrell, 2007) and cognitive development (Nelson et al., 2014; Ruggeri et al., 2015).

\subsection{Probability Gain: Reducing Prediction Error}

Probability gain (PG; Baron, 1985) measures improvement in classification accuracy. It can alternately be viewed as measuring a kind of information gain (see Crupi et al., 2018) in which error entropy (classification error), rather than Shannon entropy, is used to measure uncertainty. PG values answers in accordance with how much they improve the probability of making a correct prediction, assuming that if a searcher has to guess which hypothesis is correct (e.g., which disease is present or the species 
of a biological specimen) they will select the most likely hypothesis given all information obtained so far. The prediction error, $1-\max P\left(h_{i}\right)$, provides the entropy function (Figure 2a):

$$
\operatorname{ent}_{\text {Error }}(H)=1-\max _{1 \leqslant i \leqslant n} P\left(h_{i}\right)
$$

with the corresponding epistemic utility of a query outcome defined as

$$
\begin{aligned}
u_{P G}\left(q_{j}\right) & =\max _{1 \leqslant i \leqslant n} P\left(h_{i} \mid q_{j}\right)-\max _{1 \leqslant i \leqslant n} P\left(h_{i}\right) \\
& =\left(1-\max _{1 \leqslant i \leqslant n} P\left(h_{i}\right)\right)-\left(1-\max _{1 \leqslant i \leqslant n} P\left(h_{i} \mid q_{j}\right)\right)
\end{aligned}
$$

In Figure 1, the probability of making a correct prediction before obtaining additional information is $70 \%$, assuming one picks the most likely hypothesis (species A). According to PG, querying the eye feature is more useful than querying the claw feature, as the eye feature leads to $77 \%$ classification accuracy, eu $\left(Q_{\text {eye }}=0.07\right.$ (Figure 1c). In contrast, the claw feature does not improve on the initial $70 \%$ accuracy based on the prior probabilities alone, $e u\left(Q_{e y e}\right)=0$. Thus, a reduction in uncertainty measured via Shannon entropy does not necessarily reduce prediction error.

PG has been widely used in psychology, including probabilistic multiple-cue categorization tasks (Nelson et al., 2010; Meder \& Nelson, 2012; Wu et al., 2017), medical diagnostic test selection (Baron et al., 1988), and reasoning in the pseudodiagnosticity paradigm (Crupi et al., 2009). This research has also revealed important constraints on the ability of OED models to predict human behavior, namely the ways in which probability information is conveyed. In experience-based category learning with hundreds of learning trials, PG provides a robust predictive model of human query selection, compared to several other models (Nelson et al., 2010). With other presentation formats, for instance when probabilities are communicated with words-and-numbers or using visualizations of frequency information, the proportion of people searching in accordance with probability gain varies strongly, being as low as about $20 \%$ and as high as about $90 \%$ (Wu et al., 2017; where chance would be $50 \%$ ). Thus, the predictive power of OED models to account for behavior cannot be judged in isolation, but requires a precise characterization of the task circumstances, especially in relation to how environmental probabilities are conveyed.

\section{A Unifying Framework for Entropy and the Value of Information: The Sharma-Mittal-Space of Entropy Measures}

Information gain and probability gain are two prominent measures of the epistemic utility of queries, with different entropies underpinning them. However, a variety of further entropy measures exist and are being used in different fields, including the measurement of species diversity in ecology (Keylock, 2005), genetic variability in evolutionary biology (Lewontin, 1972), and applications in physics (Beck, 2009). Different entropies offer alternative ways to formalize the notion of uncertainty contained in a probability distribution, with distinct mathematical properties of interest for theory and application (Crupi \& Tentori, 2014; Nelson 2005). Analogously to Shannon and error entropy, these measures give rise to different informational utility functions, with the value of queries valued in terms of their ability to reduce alternative forms of entropy (Crupi et al., 2018).

One prominent model is Hartley (1928) entropy, which was an important pre-cursor in the development of Shannon's seminal work:

$$
\operatorname{ent}_{\text {Hartley }}(H)=\log \sum_{i=1}^{n} P\left(h_{i}\right)^{0} .
$$

Hartley entropy computes the logarithm of the number of non-zero-probability hypotheses (assuming that $0^{0}=0$ and $P\left(h_{i}\right)^{0}=1$ for all $P\left(h_{i}\right)>0$; Figure 2a). A value-of-information model based on Hartley entropy resembles Popper's (1959) method of falsification, as queries are valued by their 
ability to potentially rule out (falsify) at least one of the hypotheses considered. (Note though that ruling out any hypothesis - and not only the target hypothesis - would be equally valued in this information gain model.) If no outcome can potentially rule out a hypothesis, the query has no epistemic utility. This would be the case for the scenario in Figure 1, where no outcome yields certainty about the hypotheses.

a) Shannon, Error, Hartley, and Quadratic Entropy

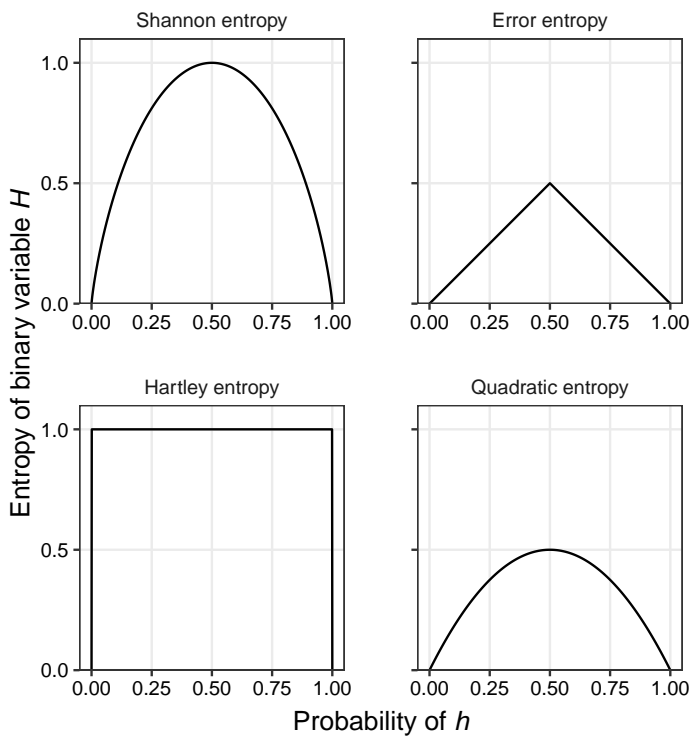

b) Sharma-Mittal Space of Entropy Measures

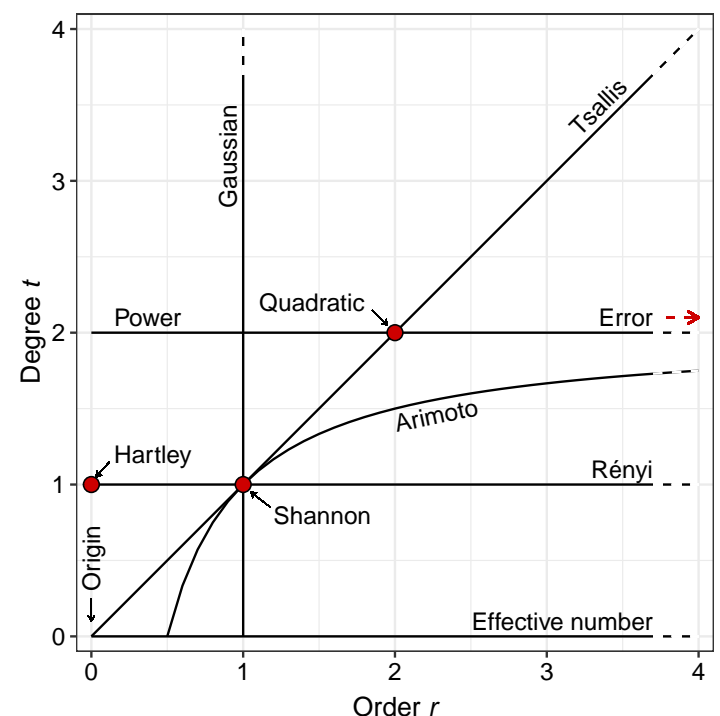

Fig. 2. Example entropy functions and the bi-parametric Sharma-Mittal family of entropy (Crupi et al., 2018). a) Examples of four prominent entropy functions for a binary hypothesis space. b) Sharma-MittalSpace with order parameter $r$ and degree parameter $t$. Each point represents a specific entropy measure (e.g., Shannon entropy); each line corresponds to a distinct generalized entropy function, such as the families of Rényi (1961) and Tsallis (1988) entropies.

Another important measure is Quadratic entropy (Vajda \& Zvárová, 2007; also known as Ginior Simpson-Gini index), formally defined as

$$
\operatorname{ent}_{\text {Quadratic }}(H)=1-\sum_{i=1}^{n} P\left(h_{i}\right)^{2} \text {. }
$$

Quadratic entropy (Figure 2a) has been widely applied in many fields, including in economics to quantify income inequality (Gini, 1921) and in biology to measure species diversity (Patil \& Taillie, 1982; Simpson, 1949). It is also frequently used in machine learning (Settles, 2010).

Different entropy measures are based on diverse conceptual and mathematical ideas about uncertainty and information. Notwithstanding these differences, several entropy measures arise as special cases in a unifying mathematical framework, the Sharma-Mittal space of entropies (SM; Sharma \& Mittal, 1975; Crupi et al., 2018). The generalized SM-entropy measure has two parameters, the order parameter $r$ and the degree parameter $t$ :

$$
\operatorname{ent}_{S M}(H)=\frac{1}{t-1}\left[1-\left(\sum_{i=1}^{n} P\left(h_{i}\right)^{r}\right)^{\frac{t-1}{r-1}}\right]
$$

The family of Sharma-Mittal entropies contains several other entropy measures as special cases (many of which exist only for well-defined limits): Shannon entropy is recovered for $r=t=1$, Hartley entropy for $r=0$ and $t=1$, Quadratic entropy for $r=t=1$, and Error entropy for $t=2$ and $r \rightarrow \infty$ 
( Figure 2b). The family of Rényi (1961) entropies is recovered for $t=1$, and the family of Tsallis (1988) entropies for $r=t$. Each of these entropy measures can be used to implement a corresponding model of the value of information that values query in accordance with the expected reduction of the chosen entropy.

In addition to providing a unified framework for quantifying entropy and uncertainty, the SharmaMittal framework offers prospects for cognitive modeling and empirical research. For instance, the logico-deductive interpretation of what constitutes rational information acquisition in Wason's (1968) selection task can be recovered in the Sharma-Mittal framework by using the reduction of Hartley entropy as a measure of epistemic utility (Crupi et al., 2018). Such analyses help to bridge seemingly incommensurate perspectives and models in a unifying mathematical framework, thereby fostering theory integration and providing guidance for empirical studies.

\section{Short- vs. Long-Run Optimality of OED Models}

OED models assess the epistemic utility of queries by considering each question's potential outcomes and their immediate implications for the hypotheses. These models were originally envisaged for situations in which only a single query can be conducted and thus they are stepwise-optimal in the sense that they optimize a particular informational utility function in the next time step. Since they disregard any future queries that could be conducted, such methods are also known as myopic or greedy models.

However, in many situations multiple queries can be conducted, for instance in medical diagnosis. In such cases, it is critical to search efficiently for information - to obtain as much information as possible with as few queries as possible (e.g., because medical tests can have intrinsic harms and are costly). Are stepwise OED models optimal in the long run when multiple queries can be conducted? The answer to this question is in the negative: Stepwise-optimal methods can provide a reasonable and tractable account for specific scenarios (Meder et al., 2019; Nelson et al., 2018), but they do not generally identify the most efficient sequence of queries-which in fact is a computationally intractable problem (Hyafil \& Rivest, 1976).
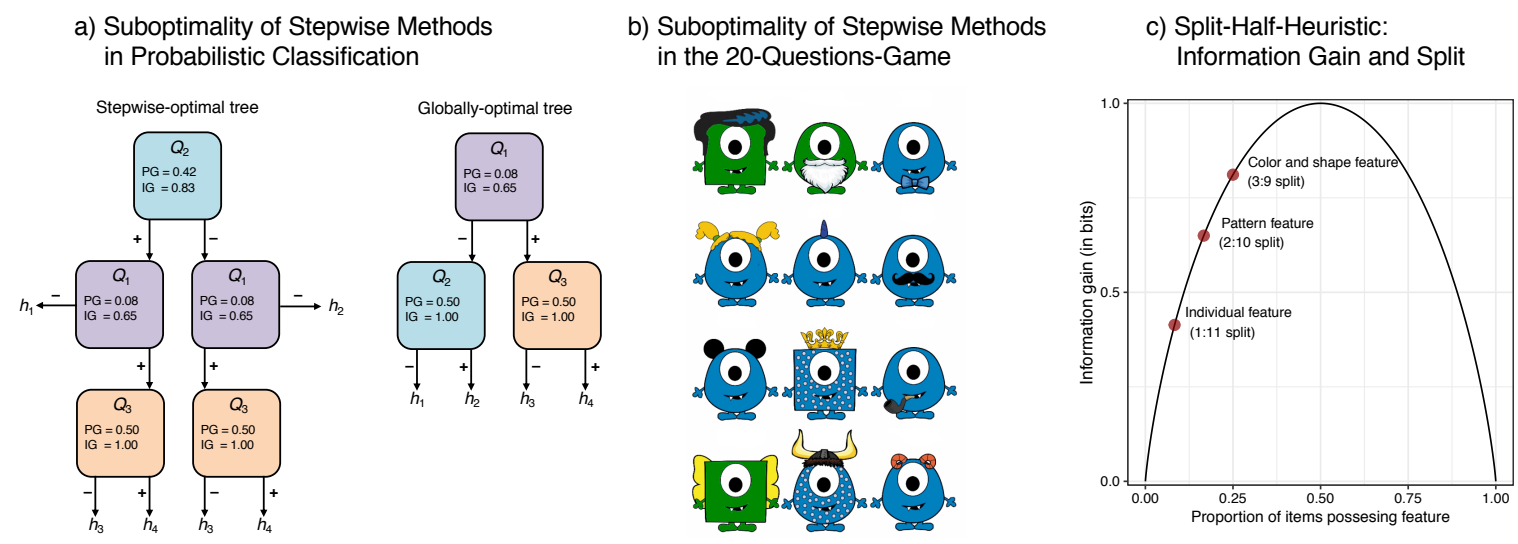

Fig. 3. Suboptimality of stepwise methods and relationships between information gain and the split-half heuristic. a) Search trees in the classification task of Meier and Blair (2013). $Q_{1}$ to $Q_{3}$ denote possible queries (binary physical features of stimuli), the $h_{i}$ denote four possible hypotheses (categories); + and - indicate the observed feature values. According to stepwise information gain (IG) and probability gain (PG), $Q_{2}$ is the most informative first query. In contrast, the globally-optimal (=most efficient) search tree starts with $Q_{1}$. b) Stimuli from a 20-questions game where stepwise-optimal methods fail to identify the most efficient first query (Meder et al., 2019). c) Relation between the split induced by a query and its stepwise information gain.

The fact that what is in some sense optimal for a one-shot search decision can be distinctly suboptimal when multiple queries can be conducted, has important implications for the theoretical and empirical analysis of human sequential search. For instance, stepwise models correspond to a 
valuation of individual queries, whereas planning a sequence of queries requires evaluating alternative search trees comprising multiple queries. However, in stark contrast to other domains such as rewardbased learning (Bellman, 1957; Sutton \& Barto, 1998), there is little research on the conflict between short- and long-term optimality and people's ability to search efficiently in situations where stepwise methods are distinctly suboptimal.

An exception is Meier and Blair (2013), who used a probabilistic classification task with four hypotheses and three (binary) queries, where the most informative first query according to several stepwise methods was not the most efficient first query in the long run (Figure 3a). Searchers were able to identify the most efficient query, but often required dozens or hundreds of learning trials to consistently select the most efficient feature first. These results show that people can learn from experience to search efficiently and overcome the limitations of stepwise approaches. However, further research is needed to achieve a better understanding of the interplay between learning processes, normative constraints imposed by sequential search scenarios, and the cognitive processes underlying query selection when stepwise methods are suboptimal.

The limitations of stepwise methods also apply in simpler situations with uniform priors over the hypotheses and deterministic likelihoods. Figure 3b shows a sequential search scenario based on the 20-questions game (Meder et al., 2019), an experimental paradigm widely used in developmental and cognitive psychology to investigate sequential search in children and adults. The goal is to identify a randomly chosen hypothesis (here: monster) by asking as few yes-no questions about its features as possible (e.g., "Is the monster blue?"). Through a series of questions searchers move from a state of maximal uncertainty with equiprobable hypotheses to knowing the true hypothesis with certainty.

A common benchmark on this task is stepwise information gain (Kachergis et al., 2017; Nelson et al., 2014; Ruggeri et al., 2016, 2017). However, selecting questions according to their maximal stepwise information gain does not necessarily entail conducting as few queries as possible. For instance, in the task environment shown in Figure 3b, the color and shape feature tie for the maximum information gain query, but starting with the color feature is more efficient in the long run, because it allows for more informative queries on subsequent steps (Meder et al., 2019). Thus, similar to Meier and Blair (2013), selecting queries in accordance with the highest stepwise expected reduction of Shannon entropy (as well as various other entropy models) fails to identify the most efficient first query. Interestingly, in this task both children and adults show very limited sensitivity to long-run considerations, with behavior better accounted for by stepwise models (Meder et al., 2019). One explanation is that learning from experience is required to search efficiently across multiple queries. Another possibility is the use of heuristic methods that may somehow correspond to stepwise OED models.

The fact that selecting queries in accordance with stepwise methods does not ensure long-run efficiency is a critical issue for both the theoretical and empirical analysis of human information acquisition, because it entails that stepwise OED models do not generally provide adequate benchmarks for evaluating people's search efficiency. While globally-optimal solutions are generally unattainable due to the quickly increasing computational complexity, for smaller numbers of hypotheses and queries the most efficient search tree(s) can be determined through dynamic programming, such that empirical paradigms can be constructed that dissociate between short- and long-run efficiency (Meder et al., 2019; Nelson et al., 2018). Such paradigms also enable contrasting stepwise and multistep models of information acquisition, which can be more efficient because they plan multiple queries ahead (Nelson et al., 2018). To what extent multistep approaches can predict human sequential search is an important open question for developing a comprehensive theory of human sequential search.

\section{From Heuristics to OED Models and Back}

In cognitive science, OED models often serve as normative benchmarks that specify the problem faced by the searcher and the quantities required to solve the task from a computational point of view. Descriptively, OED models are able to predict human behavior well on a wide range of tasks, raising questions about the nature of the cognitive processes underlying query selection. It could be that the brain indeed carries out the relevant computations without searchers being consciously aware of these processes (e.g., in visual search tasks that recruit a dedicated neural machinery), but typically OED methods are considered computational-level models of behavior which provide a "rational description" rather than a "rational calculation" (Chater et al., 2003).

One promising approach to bridge different levels of analysis is to consider heuristic strategies that choose queries without resorting to the explicit calculation of the quantities underpinning OED 
models. In psychology, the use of heuristics is often assumed to lead to systematic errors in thinking and reasoning (Tversky \& Kahneman, 1974). In a similar vein, it has frequently been argued that human search behavior does not conform to (supposedly) normative principles and suffers from a "confirmation bias" or other peculiarities. However, research has also revealed that the performance of heuristics can be on par with more computationally-heavy models and sometimes even outperform them (Gigerenzer \& Gaissmaier, 2011). A similar picture has emerged in the psychology of human inquiry, where intriguing connections between OED models and simple heuristics for query selection have been identified.

Consider the split-half heuristic (Navarro \& Perfors, 2011; Nelson et al., 2014), which is applicable in the 20-questions game (Figure 3b). This strategy does not rely on explicitly computing entropy, but rather evaluates queries in accordance with how they split the current set of hypotheses (items). According to the split-half heuristic, the closer a query comes to inducing a 50:50 split, the more informative it is. In Figure 3b, color and shape would be considered the most informative queries, as they induce a more even split (3:9) than the pattern feature (2:10) or querying individual monsters (1:11). Several papers have reported that human searchers frequently rely on this strategy, with the propensity to select features in accordance with the evenness of the induced split increasing with age (e.g., Denney \& Denney, 1973; Mosher \& Hornsby, 1966; Siegler, 1977).

How does the split-half heuristic relate to OED theories? It turns out that in the 20-questions game the stepwise information gain of a query is a direct function of the induced split: The closer the split comes to 50:50, the higher the information gain of the query (Figure 3c). Thus, a searcher using the split-half heuristic will invariably select questions in accordance with their information gain. The analytic relation between the informativeness of a query and induced split also holds for a several other entropy measures (Crupi et al., 2018) and particular non-entropic OED models (Nelson et al., 2018).

Another heuristic is the likelihood difference heuristic (Nelson, 2005; Nelson et al., 2020), which is sometimes used in binary classification tasks when probability information is conveyed through words and numbers (Slowiaczek et al., 1992). The heuristic is applicable in probabilistic search tasks with binary hypotheses and features, and values queries in accordance with the absolute difference in outcome likelihoods. Ranking queries in accordance with their likelihood difference corresponds to ranking queries in accordance with the OED model impact (Wells \& Lindsay, 1980), which for the special case of equal priors also corresponds to ranking queries according to probability gain (Nelson, 2005). In particular circumstances the likelihood difference heuristic is provably optimal for selecting queries, whereas several OED models are not (Nelson et al., 2020).

\section{Concluding Remarks: Open Questions and Challenges}

The past years have seen strong progress in building a comprehensive computational account of human inquiry based on applying OED principles to the theoretical and empirical analysis of cognition and behavior. What challenges need to be met to further advance theory and what are important venues for empirical research?

One important issue concerns the relation between informational utilities and other kinds of reward structures. For instance, in medical diagnosis the information provided by a test is of course essential, but further costs and rewards are also highly relevant (Baron \& Hershey, 1988; Nelson et al., 2020). This is because tests themselves can be intrinsically harmful (e.g., radiation) or because their outcomes have strong implications for subsequent actions (e.g., erroneously failing to quarantine an infectious person due to a false test result). While OED models evaluate queries in terms of their epistemic value, the general approach can be extended to incorporate situation-specific utilities (e.g., rewards and costs associated with decisions based upon obtained information). In this case, queries can be valued in accordance with their expected utility gain, rather than pure information value (Markant \& Gureckis, 2012; Meder \& Nelson, 2012). Incorporating other rewards and costs in the analysis of query selection might require invoking additional methods and considerations (Sharot \& Sunstein, 2020). For instance, a doctor may need to choose between a highly informative test whose results may take several days to obtain, and a less informative test whose results are immediately available. Investigating how searchers balance information value and time (i.e., temporal discounting) and how they integrate other factors (e.g., hedonic value of query outcomes and other emotions related to the anticipation of information; Li et al., 2020) are important challenges for a broader characterization of information acquisition when searchers' goals are not purely epistemic. 
A promising venue for applying the Sharma-Mittal framework to such questions is deliberate ignorance - the observation that people sometimes prefer to remain in a state of uncertainty rather than seeking out information that would resolve it (Hertwig \& Engels, 2016). People may not want to know the sex of their child before birth or prefer to not know whether they have a genetic predisposition for a disease. Such behaviors raise interesting questions for theories of human inquiry, because OED models based on entropy reduction are usually strictly non-negative, meaning that in the expectation a query can never have negative epistemic utility. The Sharma-Mittal framework, however, has the expressive power to devise models where queries can have negative information value, depending on which entropy measure is chosen to represent uncertainty and information (Crupi et al., 2018). Thus, the framework provides novel pathways to model situations where searchers consider information as contrary to their goals even in strictly informational terms and without introducing situation-specific costs.

Another key issue is the interplay between reinforcement learning and human inquiry. This is of particular importance in light of empirical findings showing that searchers can harness their learning experiences to overcome the limitations of stepwise methods (Meier \& Blair, 2013), whereas absent such experiences behavior is better accounted for by stepwise models (Meder et al., 2019). Similarly, OED models provide a much better account of human behavior when learning environmental probabilities through experience, compared to other means of conveying probability information (Meder \& Nelson, 2012; Nelson et al., 2010, Wu et al., 2017). Within the reinforcement learning framework (Sutton \& Barto, 1998), inquiry could be modeled by using informational utilities as reward functions, extending the approach from reward-based to information-based reinforcement learning ( $\mathrm{Li}$ et al., 2019). Importantly, the reinforcement learning framework offers different proposals to address the tension between short- and long-run optimality by assuming that future outcomes are temporally discounted. Stepwise OED methods could be implemented by setting the temporal discounting parameter to zero, such that only the immediate implications of query outcomes are considered. Multistep models with a longer planning horizon could be implemented by increasing the temporal discounting factor, in which case later inquiry steps are also considered (Butko \& Movellan, 2010). Integrating ideas and models from the OED and reinforcement learning literature would offer new perspectives on the learning processes and how they are constrained by OED principles.

Besides these pathways for future research, several open questions about the psychology of human inquiry and OED models exist (Coenen et al., 2019). For instance, whereas OED models require a welldefined hypothesis space and probability model, many forms of human inquiry are more open ended in that the goal is not to discriminate between existing hypotheses, but to generate hypotheses in the first place, often in the absence of explicit learning goals or situation-specific incentives (e.g., self-guided inquiry in childhood or science). While the broad goal in such cases may also be epistemic in the sense of reducing uncertainty about the world more generally, fully accounting for such forms of open-ended inquiry might require integrating OED methods with other frameworks, such as theories of curiosity (Dubey \& Griffiths, 2000). Conversely, applying OED methods can be challenging in practice, for instance in scientific experimentation when the goal is to discriminate among complex models with free parameters and the data-generating distribution is unknown (Kleinegesse \& Gutmann, 2020).

In sum, OED theories provide a computational framework for evaluating the informational value of different kinds of queries, including verbal questions, eye movements, medical tests, and experiments. The past decades have yielded substantial progress in the understanding of the computational and behavioral principles underlying human information acquisition in different domains, from neuroscience to developmental and cognitive psychology. Notwithstanding these advances and breadth of applicability, many open questions remain, and addressing these challenges will be key to making further progress toward a comprehensive theory of human information acquisition.

\section{References}

1. Austerweil, J. L., \& Griffiths, T. L. (2011). Seeking confirmation is rational for deterministic hypotheses. Cognitive Science, 35(3), 499-526. https://doi.org/10.1111/j.1551-6709.2010.01161.x

2. Baron, J. (1985). Rationality and intelligence. Cambridge, England: Cambridge University Press.

3. Baron, J., \& Hershey, J. C. (1988). Heuristics and biases in diagnostic reasoning: I. Priors, error costs, and test accuracy. Organizational Behavior and Human Decision Processes, 41(2), 259-279. https://doi. org/10.1016/0749-5978(88)90030-1

4. Beck, C. (2009). Generalised information and entropy measures in physics. Contemporary Physics, 50(4), 495-510. https://doi.org/10.1080/00107510902823517 
5. Benish, W. A. (1999). Relative entropy as a measure of diagnostic information. Medical Decision Making, 19(2), 202-206. https://doi.org/10.1177/0272989x9901900211

6. Bellman, R. (1957). Dynamic programming. Princeton, NJ: Princeton University Press.

7. Bramley, N. R., Lagnado, D. A., \& Speekenbrink, M. (2015). Conservative forgetful scholars: How people learn causal structure through sequences of interventions. Journal of Experimental Psychology. Learning, Memory, and Cognition, 41(3), 708-731. https://doi.org/10.1037/xlm0000061

8. Bruner, J. S., Goodnow, J. J., \& Austin, G. A. (1956). A study of thinking. John Wiley and Sons.

9. Butko, N. J., \& Movellan, J. R. (2010). Infomax control of eye movements. IEEE Transactions on Autonomous Mental Development, 2(2), 91-107. https://doi.org/10.1109/TAMD.2010.2051029

10. Chamberlin, T. C. (1890). The method of multiple working hypotheses. Science, 15, 92-96. https://doi. org/10.1126/science.148.3671.754

11. Chater, N., Oaksford, M., Nakisa, R., \& Redington, M. (2003). Fast, frugal, and rational: How rational norms explain behavior. Organizational Behavior and Human Decision Processes, 90(1), 63-86. https: //doi.org/10.1016/S0749-5978(02)00508-3

12. Coenen, A., Nelson, J.D. \& Gureckis, T.M. (2019). Asking the right questions about the psychology of human inquiry: Nine open challenges. Psychonomic Bulletin $\&$ Review, 26, 1548-1587. https://doi.org/ $10.3758 / \mathrm{s} 13423-018-1470-5$

13. Crupi V. (2019). Measures of biological diversity: Overview and unified framework. In E. Casetta, J. Marques da Silva, and D. Vecchi (Eds.), From assessing to conserving biodiversity (pp. 123-136). Springer

14. Crupi, V., Tentori, K., \& Lombardi, L. (2009). Pseudodiagnosticity revisited. Psychological Review, 116(4), 971-985. https://doi.org/10.1037/a0017050

15. Crupi, V., Nelson, J. D., Meder, B., Cevolani, G., \& Tentori, K. (2018). Generalized information theory meets human cognition: Introducing a unified framework to model uncertainty and information search. Cognitive Science, 42(5), 1410-1456. https://doi.org/10.1111/cogs.12613

16. Crupi, V., \& Tentori, K. (2014). State of the field: Measuring information and confirmation. Studies in History and Philosophy of Science Part A, 47, 81-90. https://doi.org/10.1016/j.shpsa.2014.05.002

17. Denney, D. R., \& Denney, N. W. (1973). The use of classification for problem solving: A comparison of middle and old age. Developmental Psychology, 9(2), 275-278. https://doi.org/10.1037/h0035092

18. Dubey, R., \& Griffiths, T. L. (2020). Reconciling novelty and complexity through a rational analysis of curiosity. Psychological Review, 127(3), 455-476. https://doi.org/10.1037/rev0000175

19. Filimon, F., Nelson, J. D., Sejnowski, T. J., Sereno, M. I., \& Cottrell, G. W. (2020). The ventral striatum dissociates information expectation, reward anticipation, and reward receipt. Proceedings of the National Academy of Sciences, 117(26), 15200-15208. https://doi.org/10.1073/pnas.1911778117

20. Gigerenzer, G., \& Gaissmaier, W. (2011). Heuristic decision making. Annual Review of Psychology, 62, 451-482. https://doi.org/10.1146/annurev-psych-120709-145346

21. Gini, C. (1921). Measurement of inequality of incomes. The Economic Journal, 31 (121), 124-126. https : //doi.org/10.2307/2223319

22. Gureckis, T. M., \& Markant, D. B. (2012). Self-directed learning: A cognitive and computational perspective. Perspectives on Psychological Science, 7(5), 464-481. https://doi .org/10.1177/1745691612454304

23. Hartley, R. V. (1928). Transmission of information. Bell System Technical Journal, 7(3), 535-563.

24. Hertwig, R., \& Engel, C. (2016). Homo Ignorans: Deliberately choosing not to know. Perspectives on Psychological Science, 11(3), 359-372. https://doi.org/10.1177/1745691616635594

25. Hyafil, L., \& Rivest, R. L. (1976). Constructing optimal binary decision trees is NP-complete. Information Processing Letters, 5(1), 15-17. https://doi.org/10.1016/0020-0190(76)90095-8

26. Kachergis, G., Rhodes, M., \& Gureckis, T. (2017). Desirable difficulties during the development of active inquiry skills. Cognition, 166, 407-417. https://doi.org/10.1016/j.cognition.2017.05.021

27. Keylock, C. J. (2005). Simpson diversity and the Shannon-Wiener index as special cases of a generalized entropy. Oikos, 109(1), 203-207. https://doi.org/10.1111/j.0030-1299.2005.13735.x

28. Klayman, J., \& Ha, Y.-W. (1987). Confirmation, disconfirmation, and information in hypothesis testing. Psychological Review, 94(2), 211-228. https://doi.org/10.1037/0033-295X.94.2.211

29. Kleinegesse, S. \& Gutmann, M.U. (2020). Bayesian experimental design for implicit models by mutual information neural estimation. Proceedings of Machine Learning Research, 119, 5316-5326.

30. Kruschke, J.K. (2008). Bayesian approaches to associative learning: From passive to active learning. Learning \& Behavior, 36, 210-226. https://doi.org/10.3758/LB.36.3.210

31. Legge, G. E., Klitz, T. S., \& Tjan, B. S. (1997). Mr. Chips: An ideal-observer model of reading. Psychological Review, 104(3), 524-553. https://doi.org/10.1037/0033-295X.104.3.524

32. Lewontin R.C. (1972) The apportionment of human diversity. In T. Dobzhansky, M. K. Hecht and W. C. Steere (Eds) Evolutionary Biology (pp. 381-398). Springer, New York, NY. https://doi.org/10.1007/ 978-1-4684-9063-3\_14

33. Li, Z., Bramley, N. R., \& Gureckis, T. M. (2020). Expectations about future learning influence momentto-moment feelings of suspense. https://doi.org/10.31234/osf.io/532tw 
34. Li, S., Sun, Y., Liu, S., Sun, Y., Gureckis, T. M., \& Bramley, N. R. (2019). Active physical inference via reinforcement learning. Proceedings of the Cognitive Science Society (pp. 2126-2132). Austin, TX: Cognitive Science Society.

35. Lindley, D. V. (1956). On a measure of the information provided by an experiment. The Annals of Mathematical Statistics, 27(4), 986-1005.

36. Lookman, T., Balachandran, P. V., Xue, D., \& Yuan, R. (2019). Active learning in materials science with emphasis on adaptive sampling using uncertainties for targeted design. npj Computational Materials, 5(1), 1-17. https://doi.org/10.1038/s41524-019-0153-8

37. Markant, D., \& Gureckis, T. M. (2012). Does the utility of information influence sampling behavior? In N. Miyake, D. Peebles, and R. P. Cooper (Eds.), Proceedings of the 34 th Annual Conference of the Cognitive Science Society (pp. 719-724). Austin, TX: Cognitive Science Society.

38. Markant, D. B., \& Gureckis, T. M. (2014). Is it better to select or to receive? Learning via active and passive hypothesis testing. Journal of Experimental Psychology: General, 143(1), 94-122. https: //doi.org/10.1037/a0032108

39. Meder, B., \& Nelson, J. D. (2012). Information search with situation-specific reward functions. Judgment and Decision Making, 7(2), 119-148. http://journal.sjdm.org/12/12314/jdm12314.pdf

40. Meder, B., Nelson, J. D., Jones, M., \& Ruggeri, A. (2019). Stepwise versus globally optimal search in children and adults. Cognition, 191, 103965. https://doi.org/10.1016/j.cognition.2019.05.002

41. Meder, B., Wu, C. M., Schulz, E., \& Ruggeri, A. (2021). Development of directed and random exploration in children. Developmental Science. Article e13095. https://doi.org/10.1111/desc.13095

42. Meier, K. M., \& Blair, M. R. (2013). Waiting and weighting: Information sampling is a balance between efficiency and error-reduction. Cognition, 126(2), 319-325. https://doi.org/10.1016/j.cognition.2012. 09.014

43. Mosher, F. A., \& Hornsby, J. R. (1966). On asking questions. In J. S. Bruner, R. R. Oliver, and P. M. Greenfield, et al. (Eds.), Studies in cognitive growth (pp. 86-102). New York, NY: Wiley.

44. Mohamed, T.P., Carbonell, J.G., \& Ganapathiraju, M.K. (2010). Active learning for human protein-protein interaction prediction. BMC Bioinformatics, 11, S57. https://doi.org/10.1186/ 1471-2105-11-S1-S57

45. Murphy, R. F. (2011). An active role for machine learning in drug development. Nature Chemical Biology, 7(6), 327-330. https://doi.org/10.1038/nchembio.576

46. Myung, J. I., \& Pitt, M. A. (2009). Optimal experimental design for model discrimination. Psychological Review, 116(3), 499-518. https://doi.org/10.1037/a0016104

47. Najemnik, J., \& Geisler, W. (2005). Optimal eye movement strategies in visual search. Nature, 434, 387-391. https://doi.org/10.1038/nature03390

48. Nakamura, K. (2006). Neural representation of information measure in the primate premotor cortex. Journal of Neurophysiology, 96(1), 478-485. https://doi.org/10.1152/jn.01326.2005

49. Navarro, D. J., \& Perfors, A. F. (2011). Hypothesis generation, sparse categories, and the positive test strategy. Psychological Review, 118(1), 120-134. https://doi.org/10.1037/a0021110

50. Nelson, J. D. (2005). Finding useful questions: On Bayesian diagnosticity, probability, impact, and information gain. Psychological Review, 112(4), 979-999. https://doi.org/10.1037/0033-295X.112.4.979

51. Nelson, J. D., \& Cottrell, G. W. (2007). A probabilistic model of eye movements in concept formation. Neurocomputing, 70, 2256-2272. https://doi.org/10.1016/j.neucom.2006.02.026

52. Nelson, J. D., \& McKenzie, C. R. M. (2009). Confirmation bias. In M. Kattan (Ed.), The Encyclopedia of Medical Decision Making (pp. 167-171). London, UK: Sage.

53. Nelson, J. D., McKenzie, C. R. M., Cottrell, G. W., \& Sejnowski, T. J. (2010). Experience matters: Information acquisition optimizes probability gain. Psychological Science, 21(7), 960-969. https://doi. org/10.1177/0956797610372637

54. Nelson, J. D., Rosenauer, C., Crupi, V., Tentori, K., \& Meder, B. (2020). On the likelihood difference heuristic and the objective utility of possible medical tests. Manuscript submitted for publication.

55. Nickerson, R. S. (1996). Hempel's paradox and Wason's selection task: Logical and psychological puzzles of confirmation. Thinking \& Reasoning, 2, 1-31. https://doi.org/10.1080/135467896394546

56. Oaksford, M., \& Chater, N. (1994). A rational analysis of the selection task as optimal data selection. Psychological Review, 101(4), 608-631. https://doi.org.10.1037/0033-295X.101.4.608

57. Oaksford, M., \& Chater, N. (1996). Rational explanation of the selection task. Psychological Review, 103(2), 381-391. https://doi.org/10.1037/0033-295X.103.2.381

58. Patil, G. P. , \& Taillie, C. (1982) Diversity as a concept and its measurement. Journal of the American Statistical Association, 77(379), 548-561. https://doi.org/10.1080/01621459.1982.10477845

59. Popper, K. R. (1959). The logic of scientific discovery. London: Hutchinson.

60. Raiffa, H., \& Schlaifer, R. O. (1961). Applied statistical decision theory. Cambridge, MA: Division of Research, Graduate School of Business Administration, Harvard University.

61. Rényi, A. (1961). On measures of entropy and information. In J. Neyman (Ed.), Proceedings of the Fourth Berkeley Symposium on Mathematical Statistics and Probability I (pp. 547-556). Berkeley, CA: University of California Press. 
62. Ruggeri, A., \& Lombrozo, T. (2015). Children adapt their questions to achieve efficient search. Cognition, 143, 203-216. https://doi.org/10.1016/j.cognition.2015.07.004

63. Ruggeri, A., Lombrozo, T., Griffiths, T. L., \& Xu, F. (2016). Sources of developmental change in the efficiency of information search. Developmental Psychology, 52(12), 2159-2173. https://doi.org/10.1037/ dev0000240

64. Savage, L. J. (1954). The foundations of statistics. New York: Wiley.

65. Schulz, E., Wu, C. M., Ruggeri, A., \& Meder, B. (2019). Searching for rewards like a child means less generalization and more directed exploration. Psychological Science, 30(11), 1561-1572. https://doi. org/10.1177/0956797619863663

66. Settles, B. (2010). Active learning literature survey. Technical Report University of Wisconsin-Madison.

67. Shannon, C. E. (1948). A mathematical theory of communication. The Bell System Technical Journal, 27, 379-423. https://doi.org/10.1002/j.1538-7305.1948.tb01338.x

68. Sharma, B., \& Mittal, D. (1975). New non-additive measures of entropy for discrete probability distributions. Journal of Mathematical Sciences, 10, 28-40.

69. Sharot, T., \& Sunstein, C.R. (2020). How people decide what they want to know. Nature Human Behaviour, 4, 14-19. https://doi.org/10.1038/s41562-019-0793-1

70. Simpson E. H. (1949). Measurement of diversity. Nature, 163, 688. https://doi.org/10.1038/163688a0

71. Skov, R. B., \& Sherman, S. J. (1986). Information-gathering processes: Diagnosticity, hypothesisconfirmatory strategies, and perceived hypothesis confirmation. Journal of Experimental Social Psychology, 103, 278-282. https://doi.org/10.1016/j.beproc.2014.01.014

72. Slowiaczek, L. M., Klayman, J., Sherman, S. J., \& Skov, R. B. (1992). Information selection and use in hypothesis testing: What is a good question, and what is a good answer? Memory 8 Cognition, 20(4), 392-405. https://doi.org/10.3758/BF03210923

73. Steyvers, M., Tenenbaum, J. B., Wagenmakers, E. J., \& Blum, B. (2003). Inferring causal networks from observations and interventions. Cognitive Science, 27, 453-489. https://doi.org/10.1016/ S0364-0213(03) 00010-7

74. Sutton, R. S., \& Barto, A. G. (1998). Reinforcement learning: An introduction. Cambridge, MA: MIT Press.

75. Tsallis, C. (1988). Possible generalization of Boltzmann-Gibbs statistics. Journal of Statistical Physics, 52(1-2), 479-487. https://doi.org/10.1007/BF01016429

76. Tversky, A., \& Kahneman, D. (1974). Judgment under uncertainty: Heuristics and biases. Science, 185(4157), 1124-1131. https://doi.org/10.1126/science.185.4157.1124

77. Vajda I. \& Zvárová J. (2007). On generalized entropies, Bayesian decisions, and statistical diversity. Kybernetika, 43(5), 675-696.

78. Wason, P. C. (1960). On the failure to eliminate hypotheses in a conceptual task. Quarterly Journal of Experimental Psychology, 12, 129-140. https://doi.org/10.1080/17470216008416717

79. Wason, P. C. (1968). Reasoning about a rule. Quarterly Journal of Experimental Psychology, 20, 273-281. https://doi.org/10.1080/14640746808400161

80. Wells, G. L., \& Lindsay, R. C. (1980). On estimating the diagnosticity of eyewitness nonidentifications. Psychological Bulletin, 88(3), 776-784. https://doi.org/10.1037/0033-2909.88.3.776

81. Wu, C. M., Meder, B., Filimon, F., \& Nelson, J. D. (2017). Asking better questions: How presentation formats influence information search. Journal of Experimental Psychology: Learning, Memory, and Cognition, 43(8), 1274-1297. https://doi.org/10.1037/xlm0000374

82. Wu, C., Schulz, E., Speekenbrink, M., Nelson, J. D., \& Meder, B. (2018). Generalization guides human exploration in vast decision spaces. Nature Human Behavior, 2, 915-924. https://doi.org/10.1038/ s41562-018-0467-4 